\title{
ARTINIAN DUO RINGS AND SELF-DUALITY
}

\author{
WEIMIN XUE \\ (Communicated by Donald S. Passman) \\ Dedicated to Professor Zhaomu Chen for his sixtieth birthday
}

\begin{abstract}
We show that the endomorphism ring of the minimal cogenerator over an Artinian duo ring is still an Artinian duo ring, and that an Artinian duo ring has self-duality if its Jacobson radical is a direct sum of colocal ideals.
\end{abstract}

Adapting Azumaya's exactness [3] to bimodules, Camillo, Fuller, and Haack [4] said that a bimodule that has a composition series whose composition factors are balanced is an exact bimodule and that a ring $R$ is an exact ring in case the regular bimodule ${ }_{R} R_{R}$ is exact.

A bimodule is called left duo (right duo) in case each left (right) submodule is a two-sided submodule and it is called $d u o$ if it is both left and right duo. Clearly a bimodule ${ }_{R} M_{S}$ is duo if and only if $R x=x S$ for all $x \in M$. A ring $R$ is called left duo (right duo, or duo) in case the bimodule ${ }_{R} R_{R}$ is left duo (right duo, or duo). Habeb [7] observed that an Artinian duo ring is exact and conjectured that the endomorphism ring of its minimal cogenerator is still an Artinian duo ring.

In this paper Habeb's conjecture is proved, and moreover we show that an Artinian duo ring $R$ has self-duality if the Jacobson radical $J(R)$ of $R$ is a direct sum of colocal ideals. In particular every Artinian duo ring with radical square zero has self-duality. These results provide further evidence for Azumaya's conjecture that exact Artinian rings have self-duality [3].

A presentation of (Morita) duality and self-duality can be found in Anderson and Fuller [1, $23, \S 24]$, or the original papers of Azumaya [2] and Morita [9]. The terminology and notations of [1] are freely used.

Courter [5, Theorem 2.2] proved that an Artinian ring $R$ with $c\left({ }_{R} R\right)=$ $c\left(R_{R}\right)$ is left duo if and only if it is right duo, where $c()$ denotes the composition length. It follows that a basic exact Artinian ring is left duo if and only if it is right duo (see [3, Corollaries 3 and 5]). We dualize Courter's result in some sense.

Received by the editors Febrary 5, 1988 and, in revised form, May 4, 1988.

1980 Mathematics Subject Classification (1985 Revision). Primary 16A49, 16A35, 16A15.

Key words and phrases. Morita duality, Artinian duo rings. 
Proposition 1. Let $R$ be a left Artinian ring with duality induced by ${ }_{R} E_{S}$ such that $c\left({ }_{R} E\right)=c\left(E_{S}\right)$. The following are equivalent:

(1) $R$ is left duo;

(2) ${ }_{R} E_{S}$ is right duo;

(3) ${ }_{R} E_{S}$ is left duo;

(4) $S$ is right duo.

Proof. (2) $\Rightarrow(3)$. Let $x \in E$, then $R x \subseteq x S \leq{ }_{R} E_{S}$. Let $(R x)^{*}=$ $\operatorname{Hom}_{R}(R x, E)$ and $x(R x)^{*}=\left\{(x) f \mid f \in(R x)^{*}\right\}$. Since ${ }_{R} E$ is injective, $x(R x)^{*} \subseteq x S$. On the other hand, $x s=(x)\left(\left.s\right|_{R x}\right) \in x(R x)^{*}$ for all $s \in S$. Hence, $x(R x)^{*}=x S$, and we have

$$
c\left({ }_{R} R x\right)=c\left((R x)_{S}^{*}\right)=c\left(x(R x)_{S}^{*}\right)=c\left(x S_{S}\right)=c\left(_{R}(x S)\right),
$$

where the second equality holds since there is an isomorphism $(R x)_{S}^{*} \cong x(R x)_{S}^{*}$ given by $f \mapsto(x) f$, and the last equality holds since ${ }_{R} E_{S}$ is right duo, $x S \leq$ ${ }_{R} E_{S}$ and $c\left({ }_{R} E\right)=c\left(E_{S}\right)$. Thus it follows that $R x=x S$.

Similarly we can prove (3) $\Rightarrow(2)$, and (1) $\Leftrightarrow(2)$ and $(3) \Leftrightarrow(4)$ follow from [1, Theorem 24.5].

As Habeb [7] noted, an Artinian duo ring is a direct sum of local subrings. Let $R$ be a local Artinian duo ring. Habeb [7] proved that if $R / J(R)$ is noncommutative then $R$ is uniserial, and that if $J(R)^{2}=0$ then $S=\operatorname{End}\left({ }_{R} E(R / J(R))\right)$ is a local Artinian duo ring with radical square zero. He conjectured that $S$ is an Artinian duo ring without assuming $J(R)^{2}=0$. The next result answers this conjecture positively.

Theorem 2. If $R$ is an Artinian duo ring with minimal cogenerator ${ }_{R} E$ and $S=\operatorname{End}\left({ }_{R} E\right)$, then ${ }_{R} E_{S}$ is duo and $S$ is an Artinian duo ring.

Proof. The hypothesis implies that the exact bimodule ${ }_{R} E_{S}$ defines a duality and that $R$ and $S$ are basic exact Artinian rings (see [3,7]). Then $c\left({ }_{R} E\right)=$ $c\left(E_{S}\right)$ and $c\left({ }_{S} S\right)=c\left(S_{S}\right)$ by [4, Lemma 2.1]. Now the results follow from Proposition 1 and [5, Theorem 2.2].

A module ${ }_{R} M$ is called local (colocal) in case $M / \operatorname{Rad}\left({ }_{R} M\right) \quad\left(\operatorname{Soc}\left({ }_{R} M\right)\right)$ is simple. Now we show that there is a large class of Artinian duo rings which do have self-duality. In particular, we prove Azumaya's conjecture for duo rings with radical square zero.

Theorem 3. If $R$ is an Artinian duo ring such that $J(R)$ is a direct sum of colocal ideals, then $R$ has self-duality.

Proof. Assume that $R$ is local. Let ${ }_{R} E=E(R / J(R))$ and $S=\operatorname{End}\left({ }_{R} E\right)$. Then ${ }_{R} E_{S}$ is duo and $S$ is a local Artinian duo ring (Theorem 2). We need to show $R \cong S$.

Since $J(R)$ is a direct sum of colocal ideals, it follows that $E_{S} / \operatorname{Soc}\left(E_{S}\right)$ is a direct sum of local submodules. So there are $x_{1}, \cdots, x_{n} \in E$ such that

$$
E / \operatorname{Soc}\left(E_{S}\right)=\oplus_{i=1}^{n} \bar{x}_{i} S=\oplus_{i=1}^{n} R \bar{x}_{i} .
$$


And then

$$
E=\sum_{i=1}^{n} x_{i} S=\sum_{i=1}^{n} R x_{i}
$$

from the fact that $R$ local implies $\operatorname{Soc}\left(E_{S}\right) \subseteq x_{i} S$, for all $i$. We may assume that $n \geq 2$. (If $n=1, R$ is $Q F$.) Using results in [1,§24] we have

so

$$
\begin{aligned}
r_{E}\left(l_{R}\left(x_{i}\right)+\left(\bigcap_{j \neq i} l_{R}\left(x_{j}\right)\right)\right) & =r_{E} l_{R}\left(x_{i}\right) \cap\left(\sum_{j \neq i} r_{E} l_{R}\left(x_{j}\right)\right) \\
=x_{i} S \cap\left(\sum_{j \neq i} x_{j} S\right) & =\operatorname{Soc}\left(E_{S}\right)=\operatorname{Soc}\left({ }_{R} E\right)=r_{E}(J(R)),
\end{aligned}
$$

Similarly, we have

$$
J(R)=l_{R}\left(x_{i}\right)+\left(\bigcap_{j \neq i} l_{R}\left(x_{j}\right)\right), \quad i=1, \cdots, n .
$$

$$
J(S)=r_{S}\left(x_{i}\right)+\left(\bigcap_{j \neq i} r_{S}\left(x_{j}\right)\right), \quad i=1, \cdots, n .
$$

Now since $R x_{i}=x_{i} S$, we have a ring isomorphism

$$
\phi_{i}: R / l_{R}\left(x_{i}\right) \cong S / r_{S}\left(x_{i}\right)
$$

given by

$$
\phi_{i}: \bar{r} \mapsto \bar{s} \quad \text { if } r x_{i}=x_{i} s .
$$

So we get a ring isomorphism

And since

$$
\phi=\oplus_{i} \phi_{i}: \oplus_{i} R / l_{R}\left(x_{i}\right) \cong \oplus_{i} S / r_{S}\left(x_{i}\right) .
$$

$$
\bigcap_{i} l_{R}\left(x_{i}\right)=l_{R}\left(\sum_{i} x_{i} S\right)=l_{R}(E)=0,
$$

we have a canonical embedding of rings

$$
f: R \hookrightarrow \oplus_{i} R / l_{R}\left(x_{i}\right)
$$

Similarly, we have a canonical embedding of rings

$$
g: S \hookrightarrow \oplus_{i} S / r_{S}\left(x_{i}\right)
$$

To show $R \cong S$, we only need to show that

$$
\phi(\operatorname{Im}(f))=\operatorname{Im}(g) .
$$

We shall show $\phi(\operatorname{Im}(f)) \subseteq \operatorname{Im}(g)$; the other containment can be proved similarly. To do so, we show that for each $r \in R$ there is an $s \in S$, such that

$$
r x_{i}=x_{i} s, \text { for all } i \text {. }
$$

Let $r \in R$, and we define an $s \in S=\operatorname{End}\left({ }_{R} E\right)$ by

$$
s: \sum_{i} r_{i} x_{i} \mapsto \sum_{i} r_{i} r x_{i}, \quad \text { for } \sum_{i} r_{i} x_{i} \in{ }_{R} E=\sum_{i} R x_{i} \text {. }
$$


If $\sum_{i} r_{i} x_{i}=0$, each $r_{i} \in J(R)=l_{R}\left(x_{i}\right)+\cap_{j \neq i} l_{R}\left(x_{j}\right)$ again by the assumption that $R$ is local, and hence, $r_{i}=y_{i}+z_{i}$ for some $y_{i} \in l_{R}\left(x_{i}\right)$ and $z_{i} \in$ $\cap_{j \neq i}\left(l_{R}\left(x_{j}\right)\right)$. Since each $l_{R}\left(x_{i}\right)$ is an ideal of $R$, we have

$$
\begin{aligned}
& \sum_{i} r_{i} r x_{i} \\
& \quad=\sum_{i}\left(y_{i}+z_{i}\right) r x_{i}=\sum_{i} z_{i} r x_{i} \\
& =\left(\sum_{j} z_{j}\right) r\left(\sum_{i} x_{i}\right)=\left(\sum_{j} z_{j}\right)\left(\sum_{i} x_{i}\right) s^{\prime} \\
& =\left(\sum_{i} z_{i} x_{i}\right) s^{\prime}=\left(\sum_{i}\left(y_{i}+z_{i}\right) x_{i}\right) s^{\prime}=\left(\sum_{i} r_{i} x_{i}\right) s^{\prime}=0,
\end{aligned}
$$

where $r\left(\sum_{i} x_{i}\right)=\left(\sum_{i} x_{i}\right) s^{\prime}$ for some $s^{\prime} \in S$ since ${ }_{R} E_{S}$ is duo. Hence, $s$ is well defined, and then $s \in S$. By the definition of $s$, we have $r x_{i}=x_{i} s$ for all $i$.

Corollary 4. If $R$ is an Artinian duo ring with $J(R)^{2}=0$, then $R$ has selfduality.

A ring $R$ with ideal $X$ and subring $D$ such that $X^{2}=0$ and $R=D \oplus X$ is called a trivial extension (see [4], for example) of $D$ by $X$. The usual notation for this is $R=D \propto X$. If ${ }_{D} X_{D}$ is a bimodule then one constructs such a ring from $D \times X$ with multiplication $\left(d_{1}, x_{1}\right)\left(d_{2}, x_{2}\right)=\left(d_{1} d_{2}, d_{1} x_{2}+x_{1} d_{2}\right)$. All trivial extensions of commutative semisimple rings have self-duality (see $[4, \mathrm{p}$. 51]), so recalling Habeb's observation that nonserial local Artinian duo rings are commutative modulo the radical, we conclude with an example showing that the rings of Theorem 3 need not be trivial extensions of commutative semisimple rings.

In [8], Mano gave a local uniserial ring as follows:

Let $F$ be a field and let $K=F(X)$ be the field of rational functions over $F$ with formal derivative ()$^{\prime}$. Put

$$
A=K \times K \times K
$$

as Abelian group. For $\left(k_{1}, k_{2}, k_{3}\right),\left(l_{1}, l_{2}, l_{3}\right) \in A$, define

$$
\left(k_{1}, k_{2}, k_{3}\right)\left(l_{1}, l_{2}, l_{3}\right)=\left(k_{1} l_{1}, k_{1} l_{2}+k_{2} l_{1}, k_{1} l_{3}+k_{2} l_{2}+k_{3} l_{1}+k_{1}^{\prime} l_{2}\right) \text {. }
$$

Then $A$ is a noncommutative local uniserial ring of length 3 such that $A / J(A)^{2}$ is commutative.

Example 5. Let $A$ be a local uniserial ring of length $n \geq 2$ with $A / J(A)^{n-1}$ commutative (e.g., the ring $A$ given above). Let $J=J(A)$ and $R=A \propto$ $\left(J / J^{2}\right)$. Then $R$ is a local Artinian duo ring: Since $A$ is a local uniserial ring, $J=A p=p A$ for some $p \in J \backslash J^{2}$ and then $0 \neq \bar{p}=p+J^{2} \in J / J^{2}$. Let $(a, b \bar{p}) \in R$. If $a \in A \backslash J$, then $(a, b \bar{p})$ is a unit of $R$ and $R(a, b \bar{p})=R=$ 
$(a, b \bar{p}) R$; so let $(a p, b \bar{p}) \in R$, then for $(c, d \bar{p}) \in R$, we have

$$
\begin{aligned}
(a p, b \bar{p})(c, d \bar{p}) & =(a p c, b \bar{p} c) \\
& =\left(a c_{1} p, b c_{1} \bar{p}\right) \\
& =\left(c_{1} a p, c_{1} b \bar{p}\right) \quad\left(\text { since } A / J^{n-1}\right. \text { is commutative) } \\
& =\left(c_{1}, 0\right)(a p, b \bar{p}) \in R(a p, b \bar{p}),
\end{aligned}
$$

where $p c=c_{1} p$ for some $c_{1} \in A$ since $A p=p A$. So we have $(a p, b \bar{p}) R \subseteq$ $R(a p, b \bar{p})$. The other containment can be proved similarly, and so $R$ is duo. Now

$$
J(R)=J \times\left(J / J^{2}\right)=(J \times 0) \oplus\left(0 \times J / J^{2}\right),
$$

where $J \times 0$ is a uniserial ideal and $0 \times J / J^{2}$ is a simple ideal of $R$. Hence, this ring $R$ has self-duality by Theorem 3. If $A$ is not commutative, neither is $R$ since $R /\left(0 \times J / J^{2}\right) \cong A$ as rings. And if $n>2$, then $R$ is not a trivial extension of semisimple rings since $J(R)^{2} \neq 0$ in this case.

Dischinger and Müller [6] and Waschbüsch [10] proved that Artinian serial rings (which are exact according to [3]) have self-duality. The above example is not covered by this theorem, since it is not serial. However this example is a trivial extension of a serial ring.

\section{ACKNOWLEDGMENT}

This paper will be a portion of a Ph.D. thesis written under the supervision of Professor Kent R. Fuller and submitted to the graduate faculty of the University of Iowa. The author wants to express his sincere gratitude to Professor Fuller for his encouragement and invaluable advice.

\section{REFERENCES}

1. F. W. Anderson and K. R. Fuller, Rings and categories of modules, Springer-Verlag, Berlin, 1974.

2. G. Azumaya, A duality theory for injective modules, Amer. J. Math. 81 (1959), 249-278.

3. __ Exact and serial rings, J. Algebra 85 (1983), 477-489.

4. V. P. Camillo, K. R. Fuller, and J. K. Haack, On Azumaya's exact rings, Math. J. Okayama Univ. 28 (1986), 41-51.

5. R. C. Courter, Finite dimensional right duo algebras are duo, Proc. Amer. Math. Soc. 84 (1982), 157-161.

6. F. Dischinger and W. Müller, Einreihig zerlegbare artinsche ringe sind selbstdual, Arch. Math. 43 (1984), 132-136.

7. J. M. Habeb, On Azumaya's exact rings and Artinian duo rings, Ph.D. thesis, Indiana University, 1987.

8. T. Mano, Uniserial rings and skew polynomial rings, Tokyo J. Math. 7 (1984), 209-213.

9. K. Morita, Duality for modules and its applications to the theory of rings with minimum condition, Tokyo Kyoiku Daigaku, Ser A 6 (1958), 83-142.

10. J. Waschbüsch, Self-duality of serial rings, Comm. Algebra 14 (1986), 581-589.

Department of Mathematics, The University of Iowa, Iowa City, Iowa 52242 